\title{
Light-hole exciton mixing and dynamics in Mn-doped quantum dots
}

\author{
V. Moldoveanu, ${ }^{1}$ I. V. Dinu, ${ }^{1}$ R. Dragomir, ${ }^{1,2}$ and B. Tanatar ${ }^{3}$ \\ ${ }^{1}$ National Institute of Materials Physics, P.O. Box MG-7, Bucharest-Magurele, Romania \\ ${ }^{2}$ Faculty of Physics, University of Bucharest, Romania \\ ${ }^{3}$ Department of Physics, Bilkent University, Bilkent 06800, Ankara, Turkey
}

(Received 12 February 2016; revised manuscript received 4 April 2016; published 18 April 2016)

\begin{abstract}
We investigate theoretically the spectral and dynamical effects of the short-range exchange interaction between a single manganese $(\mathrm{Mn})$ atom hosted by cylindrical CdTe quantum dots and its light-hole excitons or biexcitons. Our approach is based on the Kohn-Luttinger $k \cdot p$ theory and configuration interaction method, the dynamics of the system in the presence of intraband relaxation being derived from the von Neumann-Lindblad equation. The complex structure of the light-hole exciton absorption spectrum reveals the exchange-induced exciton mixing and depends strongly on the Mn position. In particular, if the Mn atom is closer to the edges of the cylinder, the bright and dark light-hole excitons are mixed by the hole-Mn exchange alone. Consequently, their populations exhibit exchange-induced Rabi oscillations which can be viewed as optical signatures of light-hole spin reversal. Similar results are obtained for mixed biexcitons, in this case the exchange-induced Rabi oscillations being damped by the intraband hole relaxation processes. The effect of light-hole heavy-hole mixing is also discussed.
\end{abstract}

DOI: 10.1103/PhysRevB.93.165421

\section{INTRODUCTION}

More than a decade ago photoluminescence spectroscopy measurements [1] revealed the six excitonic emission lines of CdTe quantum dots (QDs) doped with manganese (Mn) ions. These lines were unambiguously associated with $2 M+1$ projections on the $z$ axis of the $M n$ spin $M=5 / 2$ and provided a direct evidence of its exchange interaction with electrons and holes. Nowadays, the optically active QDs with a single magnetic dopant are intensively studied as promising solotronic devices $[2,3]$.

A series of recent experiments supports these ambitious expectations. The coherent precession of a localized Mn spin embedded in a CdTe quantum dot has been recently probed [4] and the exciton luminescence of CdTe/ZnTe QDs in the presence of a cobalt ion was observed [3]. The formation of singlet and triplet states in self-assembled heavy-hole (HH) QDs in the presence of a Mn atom has been discussed recently $[5,6]$. The experimental studies of Léger et al. [7] revealed that the in-plane QD asymmetry and Mn position competitively contribute to the level structure of the Mn "dressed" excitons and lead to very different photoluminescence spectra. Spin population trapping and optically dressed states under strong resonant excitation have been reported [8]. A study on the valence band mixing of heavy-hole excitons has been performed by Kyrychenko and Kossut [9].

Bree et al. [10] emphasized the different optical properties of Mn-doped III-V QDs (e.g., InAs) due to the acceptor character of the magnetic atom [11]. Later on Thuberg et al. [12] investigated theoretically the ultrafast light-induced dynamics in Mn-doped InAs QD.

The enhanced manipulation of the Mn spin via optical Stark effect was addressed both experimentally [13] and theoretically [14]. Varghese et al. [15] prepared a positively charged exciton and then recorded the dynamics of the Mn spin when interacting with a single hole. Quite recently, Pacuski et al. [16] tuned the exciton emission spectrum of a single Mn-doped QD embedded in a micropillar cavity to its single mode. This opens the way to photonic devices based on magnetic impurities.
The nontrivial effects of the Mn-exciton exchange interaction are rooted in the simultaneous spin-flip processes of the Mn and electron or hole spins. Typically, the fully interacting exciton states of the magnetically doped system become mixtures of "exchange-free" bright and dark excitons, i.e., exciton states calculated in the absence of exchange interaction. (For details and notation see Sec. II.) When calculated theoretically, the time-dependent populations of the latter states develop the so-called exchange-induced Rabi (EXR) oscillations. Reiter et al. [17] exploited this mechanism and simulated the optical switching of the Mn spin by optically addressing a heavy-hole exciton.

For purely HH systems the electron-Mn (e-Mn) exchange is fully responsible for the appearance of EXR oscillations. It couples bright and dark excitons with opposite electron spins [18], while the hole-Mn (h-Mn) interaction contributes only to the splitting of the six absorption peaks via the diagonal Ising part. The heavy-hole spin-flip processes are prevented by selection rules [19]. In turn, a light-hole (LH) exciton experiences both electron and hole spin flips [20]. In this case the exciton states of the QD-Mn system are mixtures of more than two exchange-free excitons, which at first glance prevents one to distinguish effects of the h-Mn exchange alone or to selectively flip the LH spin while conserving the electronic spin. The optical generation of LHs by highly focused opticalvortex beams has been theoretically investigated in a recent work [21].

In this work we consider, from a theoretical point of view, two issues which to our best knowledge were not systematically studied for Mn-doped QDs supporting lighthole excitons: (i) the role of the Mn position on the exciton or biexciton mixing as well as on absorption spectra and (ii) the h-Mn exchange-induced Rabi oscillations of LH excitons and biexcitons.

Let us note that previous studies on electronic structure [22,23] or purely $\mathrm{HH}$ systems [24] emphasized that the exchange interaction with the $\mathrm{Mn}$ atom depends strongly on its position and changes the energy spectrum. Our calculations illustrate how the position-dependent e-Mn and h-Mn exchange 
controls the light-hole exciton mixing which in turn changes the absorption spectra and the EXR oscillations. Let us stress that the Mn location can be experimentally controlled [25].

It is well known that a light-hole ground state in optically active QDs can be experimentally achieved either by tuning the aspect ratio (i.e., height/radius ratio) [26,27] or by applying tensile strain on the structure [28]. Here we take the first route and investigate QDs whose dominant light-hole character of ground-state excitons is controlled by the aspect ratio.

The rest of the paper is organized as follows: Sec. II presents the essential features of our model and formalism, Sec. III A contains the results on LH excitons, Sec. III B the results on mixed biexcitons, and the conclusions are given in Sec. IV.

\section{THE MODEL}

The starting point of our study is the calculation of the single-particle wave functions for electrons and holes confined in a cylindrical QD. To this end we use the four-band Kohn-Luttinger (KL) Hamiltonian for valence band states and the $k \cdot p$ theory. (See Ref. [29] for more details.) The confinement potential leads to the following definition of the envelope functions ( $\rho, \theta$, and $z$ are cylindrical coordinates):

$$
\phi_{m_{z} n l}(\rho, \theta, z)=\frac{e^{i m_{z} \theta}}{\sqrt{\pi} R} \frac{J_{m_{z}}\left(\alpha_{n}^{m_{z}} \rho / R\right)}{\left|J_{m_{z}+1}\left(\alpha_{n}^{m_{z}}\right)\right|} \xi_{l}(z) .
$$

Here $m_{z}$ is the orbital quantum number, $\alpha_{n}^{m_{z}}$ is the $n$th zero of the Bessel function $J_{m_{z}}$, and $\xi_{l}$ are eigenfunctions associated with the vertical confinement, that is, $\xi_{l}(z)=\sqrt{\frac{2}{H}} \sin \left(\frac{\pi l z}{H}\right)$ for $l$ even and $\xi_{l}(z)=\sqrt{\frac{2}{H}} \cos \left(\frac{\pi l z}{H}\right)$ for $l$ odd. The QD radius and height are denoted by $R$ and $H$, respectively. The hole states are linear combinations of basis vectors $\left|J_{z}, m_{z}, n, l\right\rangle:=$ $\left|\phi_{m_{z} n l}\right\rangle\left|J_{z}\right\rangle$,

$$
\left|\psi_{i}^{h}\right\rangle=\sum_{J_{z}+m_{z}=F_{z}} \sum_{n, l} C_{n, l}^{i, F_{z}}\left|\phi_{m_{z} n l}\right\rangle\left|J_{z}\right\rangle,
$$

where we introduced the total orbital quantum number $F_{z}=J_{z}+m_{z}$ and the Bloch band-edge states $\left|J_{z}\right\rangle$. Heavy (light) hole states correspond to $J_{z}= \pm 3 / 2\left(J_{z}= \pm 1 / 2\right)$. The electrons in the conduction band are described by a single-band effective mass Hamiltonian, its eigenstates $\left|\psi_{j}^{e}\right\rangle$ being described by $\left|\phi_{m_{z}^{j} n^{j} l^{j}}\right\rangle\left|S_{z}^{j}\right\rangle$ where $S_{z}= \pm 1 / 2$ is the electron spin. The energies in the conduction band are denoted by $E_{i}^{c}$ and the hole energies by $E_{j}^{h}$.

At the next step we use the single-particle functions to calculate many-body interacting configurations (excitons, biexcitons, etc.) and energies in the absence of the Mn atom. The QD is described by the Hamiltonian $\hat{H}_{0}$

$$
\begin{aligned}
\hat{H}_{0}= & \sum_{i=1}^{N_{C}} E_{i}^{c} a_{i}^{\dagger} a_{i}+\sum_{j=1}^{N_{V}} E_{j}^{h} b_{j}^{\dagger} b_{j}+\frac{1}{2} \sum_{i, j, k, l} a_{i}^{\dagger} a_{j}^{\dagger} a_{l} a_{k} V_{i j k l}^{e e} \\
& +\frac{1}{2} \sum_{i, j, k, l} b_{i}^{\dagger} b_{j}^{\dagger} b_{l} b_{k} V_{l k j i}^{h h}-\sum_{i, j, k, l} a_{i}^{\dagger} b_{l}^{\dagger} b_{j} a_{k}\left(V_{i j k l}^{e h}-V_{i j l k}^{e h}\right),
\end{aligned}
$$

written in terms of creation/annihilation operators for electrons and holes. The single-particle indices $i$ and $j$ are restricted to $N_{C}$ and $N_{V}$ in view of numerical diagonalization via the configuration interaction method. (More details are given in Sec. III.)

In Eq. (3) we singled out the direct $\left(V_{i j k l}^{e h}\right)$ and exchange $\left(V_{i j l k}^{e h}\right)$ electron-hole interactions. The latter has both shortrange and long-range components. The splitting between the two pairs of bright and dark excitons is mainly given by the short-range exchange [30], while the anisotropic (nonlocal) long-range exchange contributes to the fine structure splitting (FSS) and mixing of the bright excitons. Under strong tensile strain the anisotropic exchange interaction increases and one can switch the ground state of a QD from heavy hole to light hole [28]. For isotropic QD confinement potential the bright exciton splitting vanishes [30,31]. Moreover, for the QDs considered here the strain is assumed to be small and therefore the FSS is neglected. The calculated eigenstates and eigenvalues of $\hat{H}_{0}$ are denoted by $|v\rangle$ and $\mathcal{E}_{v}$.

A useful description of the many-body states (MBSs) $|v\rangle$ is given by the spins of the electrons and holes which occupy well-defined single-particle states. For example, the LH exciton state containing a spin-up electron in the conduction band and a light hole of spin $J_{z}=-1 / 2$ is denoted by $\left|\uparrow \Downarrow_{L}\right\rangle$. Similarly, $\left|\downarrow \Uparrow_{H}\right\rangle$ stands for a $\mathrm{HH}$ exciton of $\operatorname{spin} J_{z}=3 / 2$. We stress that if HH-LH mixing is present, $J_{z}$ denotes the dominant component in the Luttinger spinors given by Eq. (2).

Recent studies emphasized that a small built-in strain induces magnetic anisotropy [15] and a fine structure of the Mn levels. Formally this is described by the term $D_{0} \hat{M}_{z}^{2}$ and one has

$$
\left(\hat{H}_{0}+D_{0} \hat{M}_{z}^{2}\right)\left|v, M_{z}\right\rangle=\left(\mathcal{E}_{v}+D_{0} M_{z}^{2}\right)\left|\nu, M_{z}\right\rangle,
$$

where $M_{z}$ is the $z$ projection of the manganese spin $\left(M_{z}=\right.$ $\pm \frac{5}{2}, \pm \frac{3}{2}, \pm \frac{1}{2}$ ) and $\hat{M}_{z}\left|M_{z}\right\rangle=M_{z}\left|M_{z}\right\rangle$.

The short-range exchange interaction between the electron (hole) spins $\vec{S}(\vec{J})$ and the manganese spin $\vec{M}$ located at $\mathbf{R}_{\mathrm{Mn}}$ is given by

$$
\begin{aligned}
H_{\mathrm{X}-\mathrm{Mn}} & =-J_{e} \vec{S} \vec{M} \delta\left(\mathbf{r}_{e}-\mathbf{R}_{\mathrm{Mn}}\right)+J_{h} \vec{J} \vec{M} \delta\left(\mathbf{r}_{h}-\mathbf{R}_{\mathrm{Mn}}\right) \\
& =H_{\mathrm{e}-\mathrm{Mn}}+H_{\mathrm{h}-\mathrm{Mn}},
\end{aligned}
$$

where $J_{e}$ and $J_{h}$ are the e-Mn and h-Mn exchange interaction strengths.

It is convenient to write down the second quantized form $\hat{H}_{\mathrm{X}-\mathrm{Mn}}$ of $H_{\mathrm{X}-\mathrm{Mn}}$ in the basis $\left\{\left|\nu, M_{z}\right\rangle\right\}$. The matrix elements of e-Mn and h-Mn exchange are found by standard calculation. For example,

$$
\begin{aligned}
\left\langle\nu, M_{z}\left|\hat{H}_{\mathrm{e}-\mathrm{Mn}}\right| v^{\prime}, M_{z}^{\prime}\right\rangle= & -J_{e} \sum_{i, j=1}^{N_{C}} \overline{\psi_{i}^{e}}\left(\mathbf{R}_{\mathrm{Mn}}\right) \psi_{j}^{e}\left(\mathbf{R}_{\mathrm{Mn}}\right) \\
& \times\left\langle S_{z}^{i} M_{z}|\overrightarrow{\hat{S}} \overrightarrow{\hat{M}}| S_{z}^{j} M_{z}^{\prime}\right\rangle\left\langle\nu\left|a_{i}^{\dagger} a_{j}\right| \nu^{\prime}\right\rangle .
\end{aligned}
$$

The product $\vec{S} \vec{M}$ in Eq. (6) contains spin-flip operators $\hat{S}_{\mp} \hat{M}_{ \pm}$ which mix the exchange-free states $\left|v, M_{z}\right\rangle$, and the Ising part $\hat{S}_{z} \hat{M}_{z}$ which would simply turn each state $|\nu\rangle$ into a manifold $\left|v, M_{z}\right\rangle$ with a fine structure controlled by $J_{e}$. Note that the matrix elements of the exchange interaction depend 
explicitly on the electronic wave functions evaluated at the Mn position. The hole-Mn exchange acquires a more complicated expression because a given Luttinger spinor is generally a combination of $\mathrm{LH}$ and $\mathrm{HH}$ states. Consequently, for a pair of states $\left\{J_{z}, J_{z}^{\prime}\right\}$ the matrix elements $\left\langle J_{z} M_{z}|\overrightarrow{\hat{J}} \overrightarrow{\hat{M}}| J_{z}^{\prime} M_{z}^{\prime}\right\rangle$ must be multiplied by their weights in the Luttinger spinor.

The total Hamiltonian of the QD-Mn system finally reads as

$$
\hat{H}=\hat{H}_{0}+D_{0} \hat{M}_{z}^{2}+\hat{H}_{\mathrm{e}-\mathrm{Mn}}+\hat{H}_{\mathrm{h}-\mathrm{Mn}} .
$$

The eigenstates of $\hat{H}$ can be written as

$$
|p\rangle=\sum_{v, M_{z}} A_{v, M_{z}}^{p}\left|v, M_{z}\right\rangle
$$

where the coefficients $A_{v, M_{z}}^{p}$ are found by diagonalization. For further use we introduce the weight of the exchange-free state $\left|v, M_{z}\right\rangle$ in a fully interacting configuration $|p\rangle$ as $w_{v, M_{z}}^{p}:=$ $\left|A_{v, M_{z}}^{p}\right|^{2}$.

The light-matter interaction is treated classically, the corresponding Hamiltonian being

$$
\hat{V}_{R}(t)=\frac{e A_{0}(t)}{m_{0}} \sum_{i, j}\left[\left(e^{-i \omega t} S_{i j}^{+}+e^{i \omega t} S_{i j}^{-}\right) a_{i}^{*} b_{j}+\text { H.c. }\right],
$$

where $m_{0}$ is the free electron mass, $A_{0}(t)$ is the pulse envelope, and $\omega$ is its frequency. We shall denote by $E$ the electric field corresponding to the vector potential $A_{0}(t)$. For simplicity we considered real rectangular envelopes. $S_{i j}^{ \pm}$denotes the interband optical coupling matrix elements $\left(\vec{e}_{\sigma_{ \pm}}\right.$are right/left circular polarization vectors):

$$
S_{i j}^{ \pm}=\left\langle\psi_{i}^{e},\left(\vec{p} \vec{e}_{\sigma_{ \pm}}\right) \psi_{j}^{h}\right\rangle .
$$

Note that in the basis $\left\{v, M_{z}\right\}$ the operator $\hat{V}_{R}(t)$ is diagonal with respect to $M_{z}$ and off diagonal with respect to $v . S_{i j}^{ \pm}$are calculated in terms of the parameter $P=\frac{i \hbar}{m_{0}}\left\langle s, p_{x} X\right\rangle$ using known values for the Kane energy [32] $E_{p}=\frac{2}{m_{0}}\left|\left\langle s, p_{x} X\right\rangle\right|^{2}=$ $\frac{2 m_{0}}{\hbar^{2}} P^{2}$.

The dynamics of the many-body configurations under ultrashort laser pulses is derived from the density operator $\hat{\rho}$ of the system, which obeys the von Neumann-Lindblad equation,

$$
i \hbar \dot{\hat{\rho}}(t)=\left[\hat{H}+\hat{V}_{R}(t), \hat{\rho}(t)\right]+i \sum_{\lambda} \mathcal{L}_{\lambda}[\rho(t)],
$$

where the dissipative operator $\mathcal{L}_{\lambda}$ describes the intraband relaxation for electrons $(\lambda=e)$ and holes $(\lambda=h)$ and is given by $(\{$,$\} denotes the anticommutator)$

$$
\mathcal{L}_{\lambda}[\rho(t)]=\sum_{i, j=1}^{N_{\lambda}} \gamma_{i j}\left\{X_{i j}^{\lambda} \rho(t) X_{i j}^{\lambda \dagger}-\frac{1}{2}\left\{X_{i j}^{\lambda \dagger} X_{i j}^{\lambda}, \rho(t)\right\}\right\} .
$$

The relaxation rates $\gamma_{i j}$ are associated to a pair of singleparticle states in the band $\lambda$, and the jump operators are defined as $X_{i j}^{e}=a_{i}^{\dagger} a_{j}$ and $X_{i j}^{h}=b_{i}^{\dagger} b_{j}$. The spin-conserving hole relaxation is the fastest process described by the relaxation time for holes $\tau_{h}=\gamma_{i j}^{-1}$, where $i, j$ are hole levels having the same spin. Spin-conserving relaxation for electrons in the conduction band is not considered here as the numerical simulations presented in Sec. II involve only the lowest single-particle states from the conduction band. The hole spin relaxation is neglected, as it is a much slower process.

The population of a given state $\left|\nu, M_{z}\right\rangle$ is given by the diagonal matrix element $P_{v, M_{z}}:=\left\langle v, M_{z}|\hat{\rho}(t)| v, M_{z}\right\rangle$, whereas the coherence between two states is given by off-diagonal elements. The statistical average of the $z$ projection of the Mn spin is given by

$$
\left\langle\hat{M}_{z}\right\rangle=\sum_{\nu, M_{z}} M_{z}\left\langle v, M_{z}|\hat{\rho}(t)| v, M_{z}\right\rangle
$$

For a fixed initial configuration, the von NeumannLindblad equation is numerically solved on a truncated Fock subspace containing the states involved in optical transitions or spin-flip processes.

\section{RESULTS AND DISCUSSION}

In this work we consider cylindrical QDs whose singleparticle hole ground state achieves a dominant LH character by appropriate tuning of the aspect ratio $H / 2 R$. For example, we find that for $R=5 \mathrm{~nm}$ and $H=11 \mathrm{~nm}$ the highest-energy single-particle valence states of the KL Hamiltonian are up to $86 \%$ made of $s$-shell light holes $\left(F_{z}=J_{z}= \pm 1 / 2\right)$ and the next two states are mostly $(82 \%) s$-shell HH-like $\left(F_{z}= \pm 3 / 2\right)$. Due to the valence band mixing the "mostly" LH Luttinger spinor has smaller $p$-shell $\mathrm{HH}$ weights as well (e.g., a $10 \% \mathrm{HH}$ component $m=-1, J_{z}=3 / 2$ ), but the total quantum number $F_{z}=J_{z}+m_{z}$ is conserved. The existence of a $\mathrm{LH}$ ground state for tall cylindrical quantum dots is also confirmed by atomistic tight-binding calculations [27].

For our system the gap between the highest-energy LH and $\mathrm{HH}$ doublets is approximately $7 \mathrm{meV}$. It also turns out that the next hole states which could be optically coupled to the $s$-shell electronic states are located $27 \mathrm{meV}$ below the highestenergy $\mathrm{HH}$ states while the next electron states are $80 \mathrm{meV}$ above the lowest-energy $s$-shell doublet. Systematic numerical simulations show that by enlarging $N_{V}$ and $N_{C}$ the energies of the exciton or mixed biexciton states considered in this work are modified by only a few $\mu \mathrm{eVs}$ and their mixing with other configurations is negligible. Typically, a good convergence is already achieved for $N_{C}=4$ and $N_{V}=6$.

The position of the manganese atom is given in cylindrical coordinates by $\left(\rho_{\mathrm{Mn}}, \theta_{\mathrm{Mn}}, z_{\mathrm{Mn}}\right)$. Note that the center of the dot corresponds to $z_{\mathrm{Mn}}=0$ and therefore $z_{\mathrm{Mn}} \in[-H / 2, H / 2]$. From Eq. (2) one infers that if the $\mathrm{Mn}$ atom is at the dot center (i.e., $\rho_{\mathrm{Mn}}=0$ ) the $m_{z}= \pm 1$ states (i.e., $p$-shell states) do not contribute to the exchange interaction as the Bessel functions $J_{ \pm 1}$ vanish at the origin. In contrast, by shifting the magnetic impurity from the origin the exchange interaction couples $p$-shell components as well [22]. The effect of the valence-band mixing will be further discussed in Sec. III B.

In the numerical calculations we use the following values of the exchange couplings: $J_{e}=15 \mathrm{eV} \AA^{3}, J_{h}=60 \mathrm{eV} \AA^{3}$. The Luttinger parameters for CdTe are $\gamma_{1}=5.37, \gamma_{2}=1.67$, and $\gamma_{3}=1.98$. $D_{0}$ typically ranges from 0 to a few tenths of $\mu \mathrm{eV}$ for large strain values [15] and insures a slow relaxation (a few $\mu \mathrm{s})$ of the Mn spin. The numerical simulations discussed in this section were obtained for $D_{0}=6 \mu \mathrm{eV}$. We found similar results for other values of $D_{0}$. The von Neumann-Lindblad 
equation is solved by taking into account both excitons and biexcitons.

\section{A. Exchange-induced LH exciton mixing and dynamics}

Suppose that the initial state of the QD-Mn system is $\mid v=$ $\left.0, M_{z}=\frac{5}{2}\right\rangle$, where $|0\rangle$ is the QD ground state in the absence of the Mn impurity, that is, the valence band states are completely filled and the conduction band states are empty. The Mn spin can be fixed by applying a magnetic field along the $z$ axis. Note that one can turn off this magnetic field and still have stable orientation of the Mn spin due to rather long relaxation times of the Mn spin.

The exchange-free bright LH excitons $\left|\uparrow \uparrow_{L} ; 5 / 2\right\rangle$ and $\left|\downarrow \Downarrow_{L} ; 5 / 2\right\rangle$ are generated by circularly polarized $\sigma_{+}$and $\sigma_{-}$ pulses. Then, a peak corresponding to each exciton appears in the absorption spectrum associated to the exchange-free Hamiltonian in Eq. (4) (not shown). Using the Fermi golden rule one can also calculate the light-hole exciton absorption spectra of the fully interacting QD-Mn system for several positions of the Mn atom along the $z$ axis, while keeping $\rho_{\mathrm{Mn}}=0$.

Figure 1 shows that while the single-peak picture still holds for $\sigma_{+}$pulses, a multiple-peak structure develops for $\sigma_{-}$-polarized light.

More precisely, instead of a single peak the $\sigma_{-}$absorption spectrum displays four peaks for $z_{\mathrm{Mn}}=0$ (dot center) and $z_{\mathrm{Mn}}=4.56 \mathrm{~nm}$, whereas for $z_{\mathrm{Mn}}=5.14 \mathrm{~nm}$ only two peaks are visible. We also find that if the Mn atom is located even closer to the cap point $z_{\mathrm{Mn}}=5.5 \mathrm{~nm}$ a single $\sigma_{-}$peak survives, which roughly coincides with the peak associated to $\sigma_{+}$polarization (not shown). Note that the energy range covered by the four $\sigma_{-}$peaks shrinks from $1.6 \mathrm{meV}$ in Fig. 1(a) to $0.2 \mathrm{meV}$ in Fig. 1(b), while the single $\sigma_{+}$peak position does not change significantly as the Mn location varies.

We find that the complex peak structure is due to the exchange-induced mixing between four noninteracting exci-

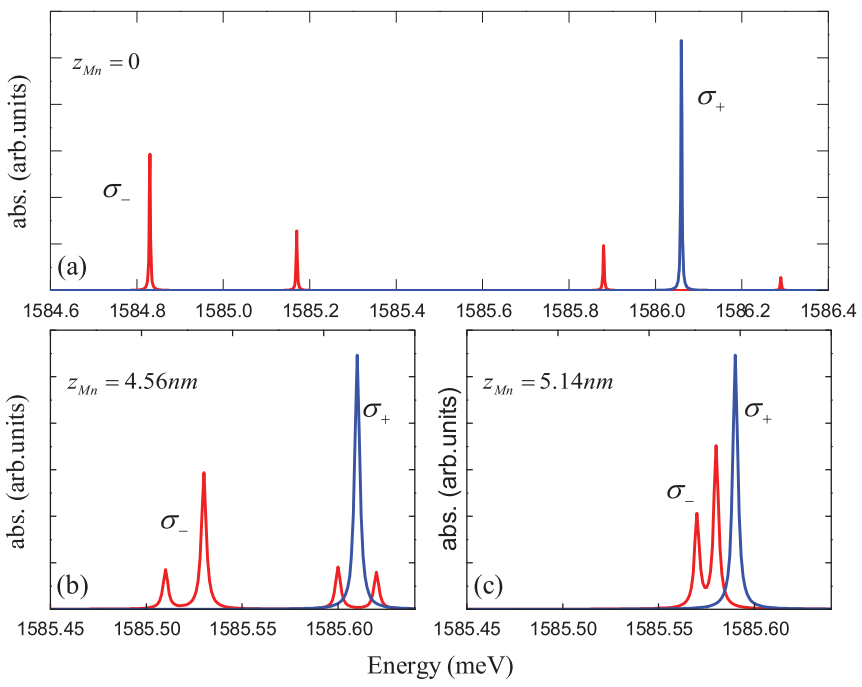

FIG. 1. Light-hole exciton absorption spectra associated to $\sigma_{-}$ (red) and $\sigma_{+}$(blue) polarized light for several positions of the $\mathrm{Mn}$ atom on the $z$ axis. (a) $z_{\mathrm{Mn}}=0$, (b) $z_{\mathrm{Mn}}=4.56 \mathrm{~nm}$, (c) $z_{\mathrm{Mn}}=5.14 \mathrm{~nm}$. The initial state of the QD is $|1,5 / 2\rangle$. tons. From Eq. (6) one can easily check that $\hat{S}_{+} \hat{M}_{-} \mid \downarrow \Downarrow_{L}$; $5 / 2\rangle=\left|\uparrow \Downarrow_{L} ; 3 / 2\right\rangle$ via e-Mn exchange and $\hat{J}_{+} \hat{M}_{-} \mid \downarrow \Downarrow_{L} ;$ $5 / 2\rangle=\left|\downarrow \Uparrow_{L} ; 3 / 2\right\rangle$ via h-Mn exchange. Moreover, the latter state pairs with the bright exciton $\left|\uparrow \uparrow_{L} ; 1 / 2\right\rangle$ due to e-Mn exchange. The mixing of the $\mathrm{LH}$ and $\mathrm{HH}$ excitons (e.g., $\left|\downarrow \Uparrow_{L} ; 3 / 2\right\rangle$ and $\left.\left|\downarrow \Uparrow_{H} ; 1 / 2\right\rangle\right)$ is negligible because of the few-meV gap between these states.

The fully interacting LH excitons can be therefore written as quadruples made of exchange-free excitons, and the corresponding absorption peaks depend on the weights of optically active exciton $\left|\downarrow \Downarrow_{L} ; 5 / 2\right\rangle$ in a given quadruple. Note, however, that the $\sigma_{+}$exciton cannot be mixed with any other LH state because $\hat{J}_{-} \hat{M}_{+}|\uparrow \uparrow ; 5 / 2\rangle=\hat{S}_{ \pm} \hat{M}_{\mp}|\uparrow \uparrow ; 5 / 2\rangle=0$. Therefore the corresponding absorption peak does not split as the Mn position changes. The peak position is in turn affected by the nonvanishing Ising part of the exchange interaction (see Fig. 1).

In Fig. 2(a) we show four LH exciton levels corresponding to quadruples made of the above-mentioned exchange-free excitons as a function of the $\mathrm{Mn} z$ coordinate, which varies
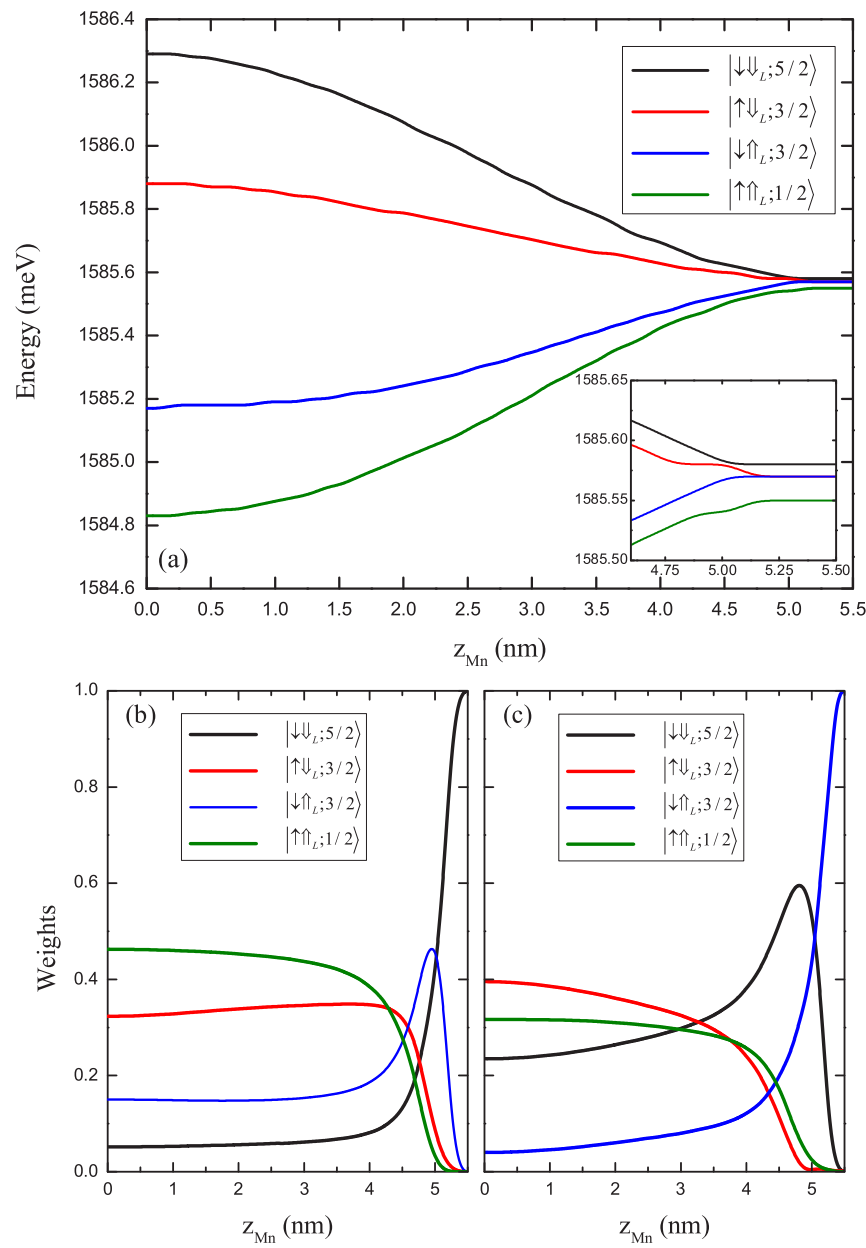

FIG. 2. (a) The energy levels corresponding to four fully interacting LH excitons as a function of the Mn position on the $z$ axis. At $z_{\mathrm{Mn}}=5.5 \mathrm{~nm}$, each level corresponds to a noninteracting exciton. (b, c) The weights of the "exchange-free" excitons for two fully interacting states as a function of Mn position. The states evolve from a strongly mixed quadruple to a single bright (b) and dark (c) exciton. 
from the cylinder center $\left(z_{\mathrm{Mn}}=0\right)$ to its top edge $\left(z_{\mathrm{Mn}}=\right.$ $5.5 \mathrm{~nm}$ ). The electron and hole envelope functions vanish on the cylinder surface so that the Mn atom and the excitons no longer interact there. Then for $z_{\mathrm{Mn}}=5.5 \mathrm{~nm}$ the levels in Fig. 2(a) correspond to exchange-free excitons and are split only by the short-range electron-hole exchange and the magnetic anisotropy term $D_{0} M_{z}^{2}$. (See the inset in Fig. 2(a).] As $z_{\mathrm{Mn}}$ approaches the cylinder center, the splitting increases and reaches a maximum at $z_{\mathrm{Mn}}=0$ because the e-Mn and h-Mn exchange interactions attain their largest values at this point. Indeed, from the explicit expression of the eigenfunction $\xi_{l}$ below [Eq. (1)] one can easily see that as $z_{\mathrm{Mn}}$ varies from zero to $\pm H / 2$ the off-diagonal matrix elements of the h-Mn interaction $\left(\left\langle\downarrow \Uparrow_{L} ; 3 / 2\left|\hat{H}_{\mathrm{h}-\mathrm{Mn}}\right| \downarrow \Downarrow_{L} ; 5 / 2\right\rangle\right)$ and e-Mn interaction $\left(\left\langle\uparrow \Downarrow_{L} ; 3 / 2\left|\hat{H}_{\mathrm{e}-\mathrm{Mn}}\right| \downarrow \Downarrow_{L} ; 5 / 2\right\rangle\right)$ decrease and eventually vanish. Note the clear correspondence between the energy levels and the $\sigma_{-}$absorption peaks in Figs. 1(a) and 1(b).

To further inspect the effect of the Mn position on the LH mixing excitons and absorption spectrum, we show in Fig. 2(b) the weights of the exchange-free excitons in the fully interacting state, which reduces to the bright exciton $\left|\downarrow \Downarrow_{L} ; 5 / 2\right\rangle$ at $z_{\mathrm{Mn}}=5.5 \mathrm{~nm}$. The associated level in Fig. 2(a) is the one with the highest energy and is clearly correlated to the rightmost $\sigma_{-}$peak in the absorption spectrum. A strong and rather constant $\mathrm{LH}$ exciton mixing is noticed as long as $z_{\mathrm{Mn}}<3.5 \mathrm{~nm}$, the dominant states being $\left|\uparrow_{\uparrow_{L}} ; 1 / 2\right\rangle$ and $\left|\uparrow \Downarrow_{L} ; 3 / 2\right\rangle$. The small amplitude of the associated absorption peak [see Fig. 1(a)] is explained by the negligible weight of the bright exciton $w_{\downarrow_{L} ; 5 / 2} \sim 0.07$. From Fig. 2(b) we notice that at $z_{M n}=5.14 \mathrm{~nm}$ the weight of the bright exciton increases to 0.60 , which leads to a higher absorption of the rightmost peak in Fig. 1(c). Moreover, Fig. 2(c) shows that the bright exciton state has a substantial weight $(\sim 0.4)$ in another fully interacting LH exciton state. [It actually corresponds to level plotted with the blue line in Fig. 2(a).] As a consequence, the amplitude of the second peak from the right in Fig. 1(c) also increases.

As $z_{\mathrm{Mn}}$ further approaches the top cylinder edge, the fully interacting state turns first to a mixture of $\left|\downarrow \Downarrow_{L} ; 5 / 2\right\rangle$ and $\left|\downarrow \Uparrow_{L} ; 3 / 2\right\rangle$ if $z_{\mathrm{Mn}} \in[4.5: 5.25] \mathrm{nm}$ and finally to the bright exciton $\left|\downarrow \Downarrow_{L} ; 5 / 2\right\rangle$. One recognizes at once that the two states are coupled by the h-Mn exchange only. The weights shown in Fig. 2(c) correspond to the LH state, which becomes dark as $z_{\mathrm{Mn}}$ approaches the top edge as $w_{\downarrow \Uparrow L ; 3 / 2}$ tends to unity in this limit.

A similar pattern is observed for the remaining two levels in Fig. 2(a), that is, for $z_{\mathrm{Mn}} \in(4.5,5.25) \mathrm{nm}$ the corresponding interacting states are mostly mixtures of $\left|\uparrow \Downarrow_{L} ; 3 / 2\right\rangle$ and $\mid \uparrow \uparrow_{L}$; $1 / 2\rangle$ (not shown). This suggests that as $z_{\mathrm{Mn}}$ approaches the cylinder edge the h-Mn interaction dominates while the e-Mn exchange plays a negligible role.

To explain this behavior one should recall that the e-Mn exchange vanishes much faster than the h-Mn coupling as a function of $z_{\mathrm{Mn}}$, since its strength $J_{e}$ is four times smaller than $J_{h}$. Moreover, the Ising terms also decrease if the Mn atom is localized near the cylinder edge. Therefore, by changing the position of the Mn atom one reaches a regime where the electron spin can hardly be reversed while the hole spin is still subjected to flip-flop processes. For $z_{\mathrm{Mn}}<4 \mathrm{~nm}$ the electron and hole spin-flip processes coexist and one cannot single out the effect of the h-Mn exchange.
As the e-Mn exchange vanishes the quadruple states turn to doublets, the two peaks in Fig. 1(c) corresponding to the linear superpositions of $\left|\downarrow \Downarrow_{L} ; 5 / 2\right\rangle$ and $\left|\downarrow \Uparrow_{L} ; 3 / 2\right\rangle$.

Motivated by previous studies of the exchange-induced Rabi oscillations for purely heavy-hole excitons [17], we also investigated the exchange-induced LH dynamics. We simulate the following two-step setup: (i) the bright $\left|\downarrow_{s} \Downarrow_{L} ; 5 / 2\right\rangle$ exciton is generated via a sharp $\sigma^{-} \pi$ pulse, provided the initial state of the system is $|1,5 / 2\rangle$; (ii) then the pulse is turned off and the populations of the exchange-free excitons change only under the e-Mn and h-Mn exchange interaction.

Figure 3 shows the populations of the exchange-free LH excitons for several positions of the Mn atom. The EXR oscillations have a much longer period than the $\sigma_{-}$pulse, and the fast decrease of the ground state $|1,5 / 2\rangle$ is not included (not shown). The oscillation amplitude depends only on the exchange interaction strength and the gap between exchange-free excitons. In the strong mixing regime $z_{\mathrm{Mn}}<$ $4.5 \mathrm{~nm}$ the exchange-induced Rabi oscillations display a messy pattern because the e-Mn and h-Mn interactions coexist (not shown) and the interacting excitons are combinations of four exchange-free states. At a formal level this means that in the strong mixing regime the system dynamics cannot be discussed in the framework of a simple three-level model (i.e., the ground state, bright and dark exciton) as it is done for $\mathrm{HH}$ systems [17]. Henceforth, we focus our analysis on the dynamics controlled mostly by the hole spin-flip processes.

Clear exchange-induced Rabi oscillations of the states $\left|\downarrow \Downarrow_{L} ; 5 / 2\right\rangle$ and $\left|\downarrow \Uparrow_{L} ; 3 / 2\right\rangle$ are obtained for $z_{\mathrm{Mn}}=5.03 \mathrm{~nm}$ in Fig. 3(b), the other two excitons having much smaller populations and a different oscillation pattern. The oscillation period is quite large (around $0.12 \mathrm{~ns}$ ), as the splitting between the bright and dark exciton levels is very small (see the inset of Fig. 2). The state of the system in this regime is a mixture of the bright and dark LH excitons. By placing the Mn atom closer to the cylinder edge, the mixing of the bright and dark

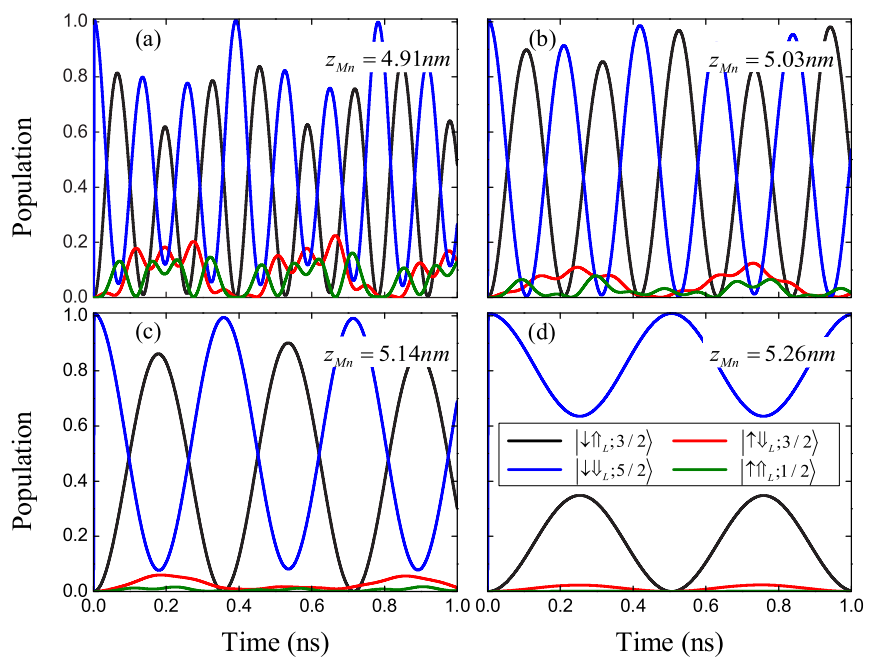

FIG. 3. Exchange-induced Rabi oscillations of populations of the four "exchange-free" excitons as a function of the Mn position: (a) $z_{\mathrm{Mn}}=4.91 \mathrm{~nm}$, (b) $z_{\mathrm{Mn}}=5.03 \mathrm{~nm}$, (c) $z_{\mathrm{Mn}}=5.14 \mathrm{~nm}$, (d) $z_{\mathrm{Mn}}=$ $5.26 \mathrm{~nm}$. The pulse that generates the bright exciton corresponds to an electric field of $E=50 \mathrm{kV} / \mathrm{cm}$. 
excitons and the amplitude of the Rabi oscillations decrease as the h-Mn interaction diminishes [see Fig. 3(d)].

From the experimental point of view, the exchange-induced Rabi oscillations can be indirectly detected using the optical transition between the bright exciton and the biexciton state $\left|\uparrow \downarrow \Uparrow_{L} \Downarrow_{L} ; 5 / 2\right\rangle$ under a $\sigma^{+} 2 \pi$ pulse. The strongest response to such a pulse is expected if it coincides with a maximum of the bright exciton population. Then the distance between consecutive higher peaks of the biexciton population roughly equals the period of the Rabi oscillations.

Let us emphasize that the exciton dynamics presented here is not affected by relaxation processes, as the optical pulse addresses only the LH ground state and the lower levels of the valence band are occupied. Note, however, that subnanosecond recombination processes would eventually dephase the exchange-induced Rabi oscillations.

The decoupling of the electron-spin-flip and hole-spin-flip processes as the $\mathrm{Mn}$ atom approaches the edge is due to the simple fact that the electron-Mn exchange interaction $J_{e}$ is four times smaller than the hole-Mn exchange $J_{h}$. This feature will hold for other confinement potentials (e.g., parabolic).

\section{B. Exchange-induced dynamics of mixed biexcitons}

The biexciton manifold we consider is built from the electronic $s$ shell and the lowest-energy LH and HH shells. As before, we start by discussing the noninteracting biexcitons. Apart from purely LH $\left(|L L\rangle:=\left|\downarrow \uparrow \Downarrow_{L} \Uparrow_{L}\right\rangle\right)$ and purely $\mathrm{HH}$ $|H H\rangle:=\left|\downarrow \uparrow \downarrow_{H} \Uparrow_{H}\right\rangle$ states one finds four "mixed" biexcitons made of both $\mathrm{LH}$ and $\mathrm{HH}$ states. We label these states by the total hole spin as follows $(D$ and $B$ stand for dark and bright): $|D+1\rangle:=\left|\downarrow \uparrow \Downarrow_{L} \Uparrow_{H}\right\rangle, \quad|D-1\rangle:=\left|\downarrow \uparrow \uparrow_{L} \Downarrow_{H}\right\rangle$, $|B+2\rangle:=\left|\downarrow \uparrow \Uparrow_{L} \Uparrow_{H}\right\rangle$, and finally, $|B-2\rangle:=\left|\downarrow \uparrow \Downarrow_{L} \Downarrow_{H}\right\rangle$.

In the presence of the $\mathrm{Mn}$ atom the optically active mixed biexcitons are coupled only by the h-Mn exchange via light-hole spin flip, as the heavy-hole spin is pinned and the e-Mn is ineffective. We shall focus our discussion on the two fully interacting states given by the superposition of $|B-2 ; 5 / 2\rangle$ and $|D-1 ; 3 / 2\rangle$. The weights of these states in the fully interacting biexciton as a function of the Mn position are presented in Fig. 4(a) (solid lines). We observe that if $z_{\mathrm{Mn}}$ is closer to the cylinder center the state is mostly dark (i.e., $\left.w_{D-1,3 / 2} \sim 0.75\right)$ and that it becomes mostly bright as $z_{\mathrm{Mn}}$ approaches the cap point.

The strongest overlap of the two states is found at $z_{\mathrm{Mn}}=2.1 \mathrm{~nm}$, although the largest value of the off-diagonal matrix element $\left\langle B-2 ; 5 / 2\left|\hat{H}_{h-\mathrm{Mn}}\right| D-1 ; 3 / 2\right\rangle$ corresponds to $z_{\mathrm{Mn}}=0$. This dependence of the biexciton mixing on the Mn position is due to the different diagonal (Ising) shifts of the bright and dark mixed biexcitons. Indeed, we find that if the Ising part of the exchange interaction is not taken into account in the calculation of the interacting biexcitons the weights of the exchange-free biexcitons do not cross [see the dashed lines in Fig. 4(a)]. Therefore the dominant state does not change as $z_{\mathrm{Mn}}$ varies, i.e., $\tilde{w}_{B-2 ; 5 / 2}>\tilde{w}_{D-1 ; 3 / 2}$ for any $z_{\mathrm{Mn}}$.

Figure 4(b) shows the biexciton levels associated with the diagonal part of the Hamiltonian in Eq. (7), whose dependence on $z_{\mathrm{Mn}}$ comes only from the Ising terms. These levels correspond to exchange-free biexcitons, so we denote
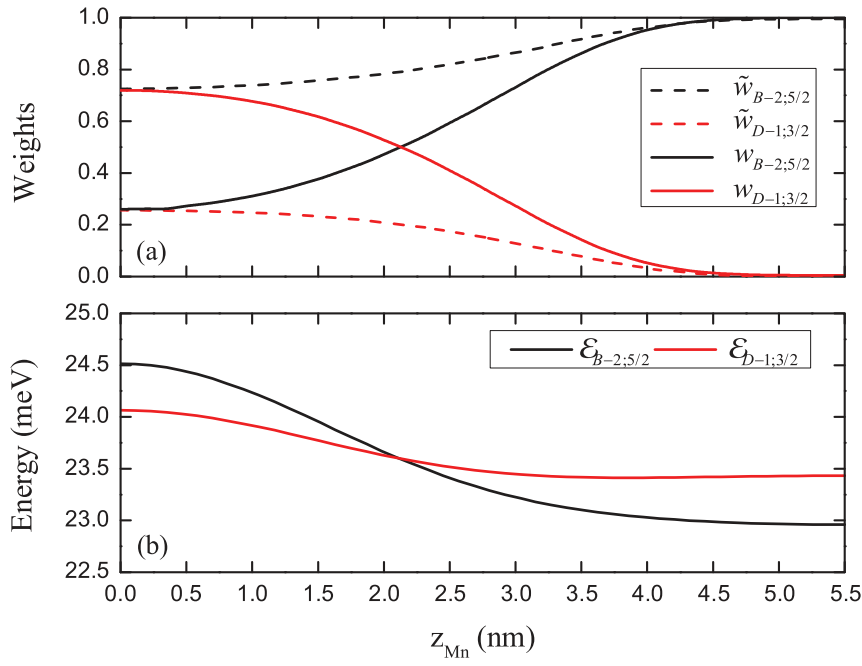

FIG. 4. (a) The weights of the bright and dark biexcitons as a function of the Mn position. The solid line weights are associated to fully interacting states, whereas the dashed lines are obtained by neglecting the Ising term in the Mn-exchange interaction. (b) The bright and dark biexciton levels in the absence of the flip-spin scattering. The level crossing is due only to the diagonal Ising term of the h-Mn exchange. Note that the degeneracy point coincides with the point of maximal mixing shown in Fig. 1(a).

them by $\mathcal{E}_{B-2 ; 5 / 2}$ and $\mathcal{E}_{D-1 ; 3 / 2}$. At $z_{\mathrm{Mn}}=5.5 \mathrm{~nm}$ the gap $\delta=\mathcal{E}_{D-1 ; 3 / 2}-\mathcal{E}_{B-2 ; 5 / 2}$ is essentially due to the hole-hole interaction and to the magnetic anisotropy term $D_{0} \hat{M}_{z}^{2}$. We find that the intraband hole-hole Coulomb interaction induces a gap of about $0.4 \mathrm{meV}$ between the bright and dark states. As the $\mathrm{Mn}$ atom approaches the center of the cylinder, the larger Ising shift pushes $\mathcal{E}_{B-2 ; 5 / 2}$ above $\mathcal{E}_{D-1 ; 3 / 2}$.

The degeneracy point $\delta=0$ corresponds to $z_{\mathrm{Mn}}=2.1 \mathrm{~nm}$ and evidently coincides with maximal biexciton mixing in Fig. 4(a).

We now investigate the exchange-induced Rabi oscillations for mixed biexcitons. To this end we introduce the following setup: (i) a sharp $\sigma_{-}$pulse generates the heavy-hole exciton $\left|\uparrow \Downarrow_{H} ; 5 / 2\right\rangle$ (notice that the electron and the hole spin of this exciton cannot be flipped by the Mn-exchange interaction), (ii) once this $\pi$ rotation is complete the pulse is switched off and one applies a second $\sigma_{-}$pulse tuned to the transition $\mid \uparrow \Downarrow_{H}$; $5 / 2\rangle \rightarrow|B-2 ; 5 / 2\rangle$. Figure 5 shows the Rabi oscillations of the bright and dark biexcitons for several positions of the $\mathrm{Mn}$ atom. The oscillation amplitude reaches a maximum at $z_{\mathrm{Mn}}=2.1 \mathrm{~nm}$ where the two biexcitons are strongly mixed [see Fig. 4(a)]. The oscillations are much faster (the period ranges from 5 to $10 \mathrm{ps}$ ) than the ones presented in the previous section, because the Coulomb gap between the mixed biexcitons is much larger than the energy spacing between the LH excitons.

We stress that the effect of the h-Mn exchange alone can be singled out from the dynamics of the mixed biexcitons irrespective of the Mn position on the $z$ axis, in contrast to the LH case where clear exchange-induced Rabi oscillations are observed only if the Mn atom is closer to the cylinder edge.

The oscillations in Fig. 5 were obtained without taking into account the intraband hole relaxation processes. The separation between the $\mathrm{LH}$ and $\mathrm{HH}$ states (i.e., the relaxation 


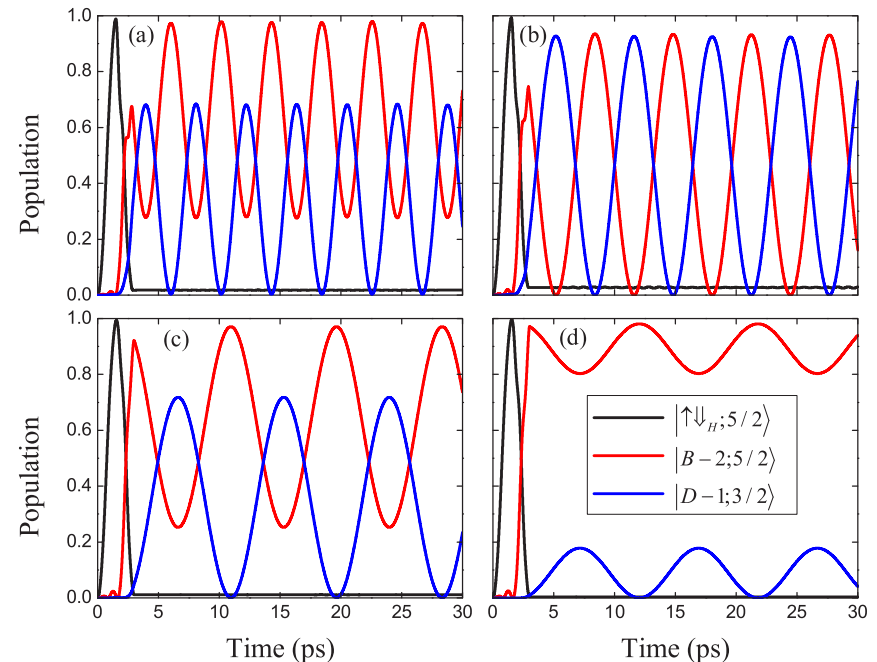

FIG. 5. Exchange-induced Rabi oscillations of bright and dark biexciton population as a function of the Mn position: (a) $z_{\mathrm{Mn}}=0$, (b) $z_{\mathrm{Mn}}=2.1 \mathrm{~nm}$, (c) $z_{\mathrm{Mn}}=3 \mathrm{~nm}$, (d) $z_{\mathrm{Mn}}=4 \mathrm{~nm}$, and $E=50$ $\mathrm{kV} / \mathrm{cm}$.

energy) is around $7 \mathrm{meV}$, which is below the frequency of the LO phonons in CdTe. (This is typically around $22 \mathrm{meV}$; see, e.g., Ref. [33].) This means that in our case the relaxation is mostly due to LA phonons. The reported values for this relaxation time range $\tau_{h}$ is from 7 to 20 ps (see, e.g., Ref. [34]).

It is easy to see that two such processes are expected to damage the exchange-induced Rabi oscillations. On one hand the $\mathrm{HH}$ exciton $\left|\uparrow \Downarrow_{H} ; 5 / 2\right\rangle$ can relax to $\left|\uparrow \Downarrow_{L} ; 5 / 2\right\rangle$; this effect can be reduced by a faster initialization of the $\mathrm{HH}$ exciton, that is, by increasing the electric field. On the other hand, the dark biexciton $|D-1 ; 3 / 2\rangle$ is depleted in favor of the purely $L H$ bright biexciton $|L L ; 3 / 2\rangle$. We find that this relaxation path is the main cause for the damping of the Rabi oscillations.

Damped Rabi oscillations of the mixed biexcitons in the presence of hole relaxation are presented in Fig. 6(a), where for simplicity we started the simulation with the fully occupied HH exciton state $\left|\uparrow \Downarrow_{H} ; 5 / 2\right\rangle$. The hole relaxation time $\tau_{h}=$ 13.3 ps. The population of the $s$-shell biexciton $|L L ; 3 / 2\rangle$ increases smoothly on each oscillation of $P_{D-1 ; 3 / 2}$ and is rather constant if the bright exciton is substantially populated. Eventually the system settles down to the $s$-shell biexciton state $(t \sim 100 \mathrm{ps})$. We notice a small population of the state $|L L ; 1 / 2\rangle$; this is due to the h-Mn interaction which couples the $\mathrm{LH}$ and $\mathrm{HH}$ spins through the transitions $|D-1 ; 3 / 2\rangle \leftrightarrows$ $|L L ; 1 / 2\rangle$. The few-meV gap between $\mathrm{LH}$ and $\mathrm{HH}$ exciton levels prevents a strong coupling and explains the small and slow occupation of $|L L ; 1 / 2\rangle . P_{L L ; 1 / 2}$ is suppressed as the $\mathrm{Mn}$ atom approaches the edge of the cylinder (not shown).

Let us stress that the rather fast switching between the mixed and $s$-shell biexciton requires both h-Mn exchange and intraband relaxation. Indeed, in the absence of the Mn atom the transition $|D-1\rangle \rightarrow|L L\rangle$ can only be achieved through hole spin-flip relaxation, which is a much slower process (i.e., nanoseconds [15]). Otherwise stated, the exchange
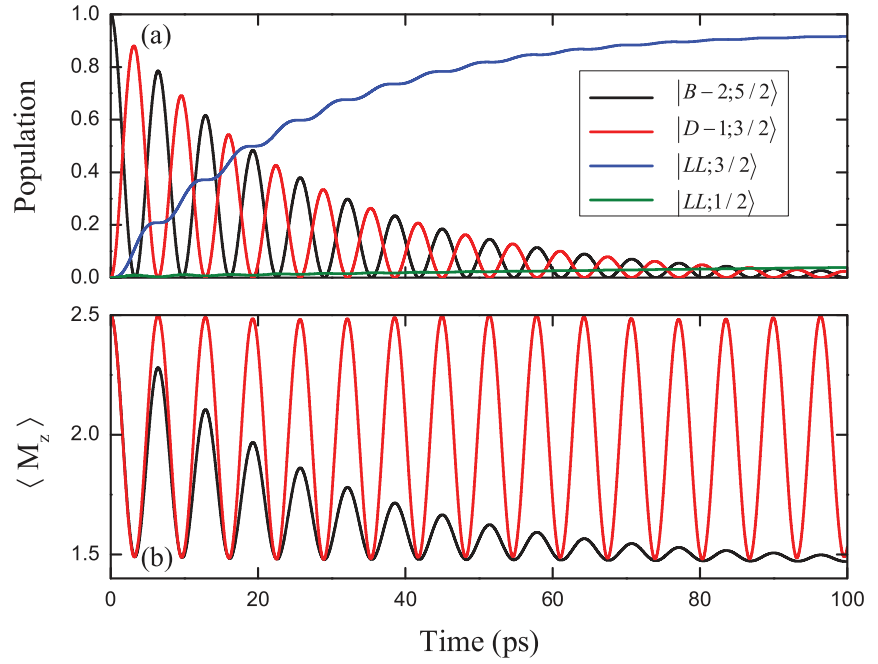

FIG. 6. (a) Damped exchange-induced Rabi oscillations of the biexciton populations in the presence of the intraband holerelaxations processes. The hole-relaxation time $\tau_{h}=13.3 \mathrm{ps}$. (b) The average $\mathrm{Mn}$ spin as a function of time for strong $\left(z_{\mathrm{Mn}}=\right.$ $2.1 \mathrm{~nm}$ ) biexciton mixing. Red line-no hole relaxation, black line $-\tau_{h}=13.3 \mathrm{ps}$, and $E=75 \mathrm{kV} / \mathrm{cm}$.

interaction with a magnetic dopant reduces the lifetime of mixed biexcitons.

The time dependence of the Mn spin is shown in Fig. 6(b). As expected, $\left\langle\hat{M}_{z}\right\rangle$ displays periodic oscillations, the largest amplitude corresponding to the maximal biexciton mixing at $z_{\mathrm{Mn}}=2.1 \mathrm{~nm}$. Similar oscillations were obtained in a threelevel model [17] in the context of all-optical manipulation of the Mn spin. However, the hole relaxation process strongly damages the oscillations and the Mn spin is eventually frozen at around $3 / 2$.

Let us finally comment on the effects of the LH-HH mixing. In order to capture such effects we performed simulations for different in-plane Mn positions where the smaller weight $p$-shell components in Luttinger spinors in Eq. (2) do not vanish. As long as $J_{ \pm 1}\left(\rho_{\mathrm{Mn}}\right) \neq 0$, these "minor" components also contribute to the exchange interaction and bring in more spin-flip processes. Consequently, the "quadruple" structure of the LH excitons holds only approximately, in the sense that other smaller weight configurations contribute as well to a fully interacting state. We find, however, that the results on distinctive LH spin-flip processes as the Mn atom approaches the cylinder edges still hold.

Figure 7 shows that the exchange-induced Rabi oscillations of the biexciton populations are still visible for the nonvanishing radial coordinate $\rho_{\mathrm{Mn}}$ (i.e., they are similar to the ones in Fig. 6). Note that the populations of the exchange-free biexcitons $|B-2 ; 5 / 2\rangle$ and $|D-2 ; 3 / 2\rangle$ do not add to unity anymore as their minor components are exchange coupled to other biexciton states with different $M_{z}$ as well. (We did not include their populations in Fig. 7.) The effect is negligible if $z_{\mathrm{Mn}}=0$ [see Figs. 7(a) and 7(b)] because the vertical confinement quantum number of the largest $p$-shell component of the "mostly" LH Luttinger spinor is even (i.e., $l=2$ ) and therefore the associated function $\xi_{l}(z)=\sqrt{\frac{2}{H}} \sin \left(\frac{\pi l z}{H}\right)$ 


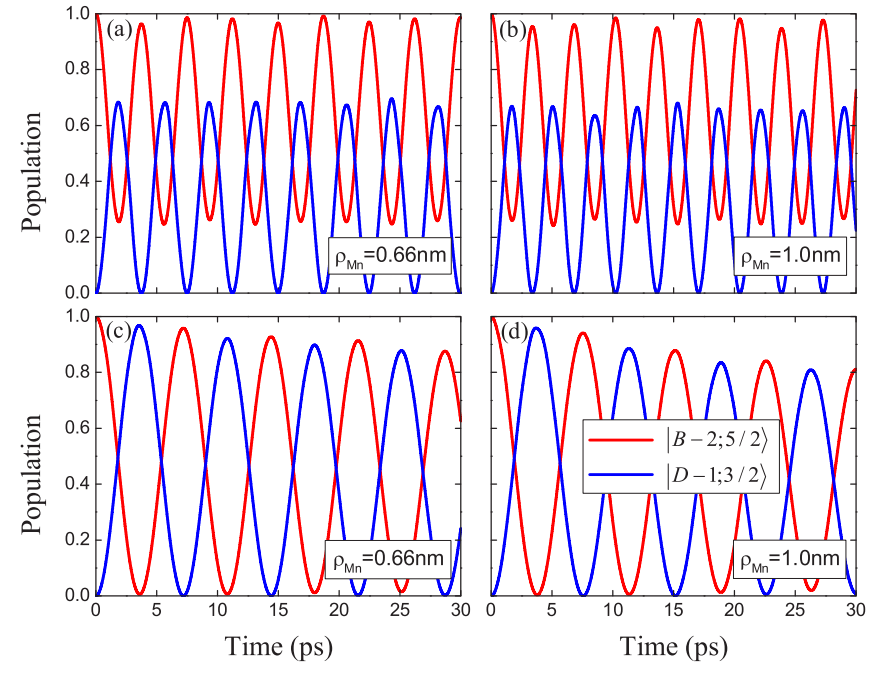

FIG. 7. The exchange-induced Rabi oscillations of the biexciton populations for different in-plane positions of the Mn atom. $(\mathrm{a}, \mathrm{b})$ $z_{\mathrm{Mn}}=0 ;(\mathrm{c}, \mathrm{d}) z_{\mathrm{Mn}}=2.45 \mathrm{~nm}$. The initial state is $|B-2 ; 5 / 2\rangle$ and the hole relaxation processes are neglected as in Fig. 5.

vanishes. This does not happen at $z_{\mathrm{Mn}}=2.45 \mathrm{~nm}$ and a more pronounced decrease of the oscillation amplitude is noticed in Figs. 7(c) and 7(d). We obtained similar results for QDs of different sizes, provided the aspect ratio $H / 2 R$ leads to "mostly" LH ground-state excitons.

\section{CONCLUSIONS}

We provide a theoretical study of the interplay of electron$\mathrm{Mn}$ and hole-Mn exchange interactions in single Mn-doped cylindrical QDs. The dependence of the exchange interaction on the manganese position is explicitly included in the numerical simulations. Its effects can be traced not only from the multiple-peak structure of the light-hole absorption spectrum, but also from the induced Rabi oscillations of the LH excitons and mixed biexcitons.

By changing the location of the manganese atom on the $z$ axis we identified two regimes of the light-hole exciton mixing. The "quadruple" regime is characterized by simultaneous e-Mn and h-Mn exchange couplings, the interacting excitons being typically made of four noninteracting states. This regime is suitable neither for the observation of the exchange-induced Rabi oscillations nor for the preparation of an exciton with a dominant light-hole spin. The second regime is achieved by placing the $\mathrm{Mn}$ atom closer to the edge of the cylinder, such that the e-Mn exchange is vanishingly small while the h-Mn interaction is still important. In this case the fully interacting LH excitons are mostly made of two noninteracting excitons which are mixed only through hole spin-flip processes. Moreover, one recovers large-amplitude Rabi oscillations between bright and dark LH excitons.

We also investigated the dynamics of the mixed biexcitons with a filled electronic $s$ shell and found hole-Mn exchangeinduced Rabi oscillations of the biexcitonic populations for any location of the Mn atom on the $z$ axis. As expected, these oscillations are damped by the intraband spin-conserving hole relaxation. The contribution of the Ising part of the exchange interaction on the biexciton mixing has been pointed out. We hope that our results motivate further experimental and theoretical investigation of LH systems.

\section{ACKNOWLEDGMENTS}

V.M., I.V.D., and R.D. acknowledge financial support from the PNCDI2 program (Grant No. PN-II-ID-PCE-20113-0091) and from Grant No. 45N/2009. B.T. acknowledges partial support from TUBITAK (112T619) and the Turkish Academy of Sciences (TUBA).
[1] L. Besombes, Y. Léger, L. Maingault, D. Ferrand, and H. Mariette, and J. Cibert, Phys. Rev. Lett. 93, 207403 (2004).

[2] P. M. Koenraad and M. E. Flatte, Nat. Mater. 10, 91 (2011).

[3] J. Kobak, T. Smoleński, M. Goryca, M. Papaj, K. Gietka, A. Bogucki, M. Koperski, J.-G. Rousset, J. Suffczyński, E. Janik, M. Nawrocki, A. Golnik, P. Kossacki, and W. Pacuski, Nat. Commun. 5, 1 (2014).

[4] M. Goryca, M. Koperski, P. Wojnar, T. Smoleński, T. Kazimierczuk, A. Golnik, and P. Kossacki, Phys. Rev. Lett. 113, 227202 (2014).

[5] A. H. Trojnar, M. Korkusinski, U. C. Mendes, M. Goryca, M. Koperski, T. Smolenski, P. Kossacki, P. Wojnar, and P. Hawrylak, Phys. Rev. B 87, 205311 (2013).

[6] U. C. Mendes, M. Korkusinski, A. H. Trojnar, and P. Hawrylak, Phys. Rev. B 88, 115306 (2013).

[7] Y. Léger, L. Besombes, L. Maingault, D. Ferrand, and H. Mariette, Phys. Rev. Lett. 95, 047403 (2005).

[8] S. Jamet, H. Boukari, and L. Besombes, Phys. Rev. B 87, 245306 (2013).

[9] F. V. Kyrychenko and J. Kossut, Phys. Rev. B 70, 205317 (2004).
[10] J. van Bree, P. M. Koenraad, and J. Fernandez-Rossier, Phys. Rev. B 78, 165414 (2008).

[11] A. Kudelski, A. Lemaitre, A. Miard, P. Voisin, T. C. M. Graham, R. J. Warburton, and O. Krebs, Phys. Rev. Lett. 99, 247209 (2007).

[12] D. Thuberg, D. E. Reiter, V. M. Axt, and T. Kuhn, Phys. Rev. B 88, 085312 (2013).

[13] C. LeGall, A. Brunetti, H. Boukari, and L. Besombes, Phys. Rev. Lett. 107, 057401 (2011).

[14] D. E. Reiter, T. Kuhn, and V. M. Axt, Phys. Rev. B 85, 045308 (2012).

[15] B. Varghese, H. Boukari, and L. Besombes, Phys. Rev. B 90, 115307 (2014).

[16] W. Pacuski, T. Jakubczyk, C. Kruse, J. Kobak, T. Kazimierczuk, M. Goryca, A. Golnik, P. Kossacki, M. Wiater, P. Wojnar, G. Karczewski, T. Wojtowicz, and D. Hommel, Cryst. Growth Des. 14, 988 (2014).

[17] D. E. Reiter, T. Kuhn, and V. M. Axt, Phys. Rev. Lett. 102, 177403 (2009).

[18] A. Manasekyan and T. Chakraborty, Nanotechnology 21, 355401 (2010). 
[19] The complete flip process of the $\mathrm{HH}$ spin $J_{z}= \pm 3 / 2$ requires a spin difference $\Delta J_{z}= \pm 3$ while the exchange-induced Mn spin flip provides only $\Delta M_{z}= \pm 1$.

[20] D. E. Reiter, T. Kuhn, and V. M. Axt, Phys. Rev. B 83, 155322 (2011).

[21] G. F. Quinteiro and T. Kuhn, Phys. Rev. B 90, 115401 (2014).

[22] F. Qu and P. Hawrylak, Phys. Rev. Lett. 95, 217206 (2005).

[23] N. T. T. Nguyen and F. M. Peeters, Phys. Rev. B 76, 045315 (2007).

[24] A. O. Govorov and A. V. Kalameitsev, Phys. Rev. B 71, 035338 (2005).

[25] K. Gietka, J. Kobak, E. Janik, J. G. Rousset, J. Borysiuk, M. Nawrocki, A. Golnik, P. Kossacki, and W. Pacuski, J. Cryst. Growth 401, 640 (2014).

[26] J. I. Climente, Solid State Commun. 152, 825 (2012).

[27] M. Zieliński, Phys. Rev. B 88, 115424 (2013).
[28] Y. H. Huo, B. J. Witek, S. Kumar, J. R. Cardenas, J. X. Zhang, N. Akopian, R. Singh, E. Zallo, R. Grifone, D. Kriegner, R. Trotta, F. Ding, J. Stangl, V. Zwiller, G. Bester, A. Rastelli, and O. G. Schmidt, Nat. Phys. 10, 46 (2014).

[29] V. Moldoveanu, I. V. Dinu, and R. Dragomir, Phys. Rev. B 89, 245415 (2014).

[30] E. Kadantsev and P. Hawrylak, Phys. Rev. B 81, 045311 (2010).

[31] C.-H. Lin, W.-T. You, H.-Y. Chou, S.-J. Cheng, S.-D. Lin, and W.-H. Chang, Phys. Rev. B 83, 075317 (2011).

[32] O. Gywat, H. J. Krenner, and J. Berezovsky, Spins in Optically Active Quantum Dots: Concepts and Methods, (Wiley-VCH, Weinheim, 2010).

[33] K. Mizoguchi, R. Morishita, and G. Oohata, Phys. Rev. Lett. 110, 077402 (2013).

[34] H. Htoon, D. Kulik, O. Baklenov, A. L. Holmes, Jr., T. Takagahara, and C. K. Shih, Phys. Rev. B 63, 241303(R) (2001). 